Groups Geom. Dyn. 9 (2015), 783-792

DOI $10.4171 /$ GGD/327
Groups, Geometry, and Dynamics

(C) European Mathematical Society

\title{
The Farrell-Jones conjecture for fundamental groups of graphs of abelian groups
}

\author{
Giovanni Gandini, ${ }^{1}$ Sebastian Meinert, ${ }^{2}$ and Henrik Rüping ${ }^{3}$
}

\begin{abstract}
We show that the Farrell-Jones conjecture holds for fundamental groups of graphs of groups with abelian vertex groups. As a special case, this shows that the conjecture holds for generalized Baumslag-Solitar groups.
\end{abstract}

Mathematics Subject Classification (2010). Primary: 18F25; Secondary: 19A31, 19B28, 19G24.

Keywords. Farrell-Jones Conjecture, algebraic K- and L-theory of group rings, fundamental groups of graphs of abelian groups, generalized Baumslag-Solitar groups, BaumslagSolitar groups.

\section{Introduction}

Denote by $\mathfrak{C}$ the class of groups that satisfy the K- and L-theoretic Farrell-Jones conjecture with finite wreath products (with coefficients in additive categories) with respect to the family of virtually cyclic subgroups. Farrell and $\mathrm{Wu}$ [5] showed that the conjecture holds for the solvable Baumslag-Solitar groups, and Wegner [9] generalized their proof to show that the conjecture in fact holds for all solvable groups.

${ }^{1}$ The first author was supported by the Danish National Research Foundation (DNRF) through the Centre for Symmetry and Deformation.

2 The second author was supported by the Deutsche Forschungsgemeinschaft (DFG) through the Berlin Mathematical School (BMS).

${ }^{3}$ The third author was supported by the Leibniz Prize of Prof. Dr. Wolfgang Lück granted by the Deutsche Forschungsgemeinschaft. 
Let $(\Gamma, \mathcal{G})$ be a finite graph of finitely generated abelian groups. We construct a group homomorphism $\phi$ from $\pi_{1}(\Gamma, \mathcal{G})$ to a semidirect product $\mathbb{Q}^{m} \rtimes F_{n}$, where $F_{n}$ denotes the free group of rank $n$. Wegner's result implies that $\mathbb{Q}^{m} \rtimes F_{n}$ lies in $\mathfrak{C}$ (Corollary 2.2). Then, given a torsion-free cyclic subgroup $C \leq \mathbb{Q}^{m} \rtimes F_{n}$ we show that its preimage $\phi^{-1}(C) \leq \pi_{1}(\Gamma, \mathcal{G})$ is a directed colimit of CAT(0)-groups and hence lies in $\mathfrak{C}$. Together with inheritance properties of $\mathfrak{C}$ (Theorem 2.1) and a sequence of colimit arguments this proves the following:

Main Theorem. Let $(\Gamma, \mathcal{G})$ be a graph of abelian groups. Then $\pi_{1}(\Gamma, \mathcal{G})$ lies in $\mathfrak{C}$.

Here, we do not require $\Gamma$ to be finite or countable, and we do not make any assumptions on the cardinality of the generating sets of the groups of $\mathcal{G}$.

A generalized Baumslag-Solitar group is the fundamental group of a finite graph of infinite cyclic groups.

Corollary 1.1. All generalized Baumslag-Solitar groups, and in particular all Baumslag-Solitar groups, lie in $\mathfrak{C}$.

Remark. Farrell and Wu have informed us about a recent independent result of theirs which proves the Farrell-Jones conjecture for Baumslag-Solitar groups.

Acknowledgements. We would like to thank Dieter Degrijse for his helpful comments.

\section{Facts about the Farrell-Jones Conjecture}

The proof of the main theorem will rely on previously known cases of the conjecture and on inheritance properties. The following list is not complete; it highlights results that will be made use of in the present paper.

Theorem 2.1 ([6],[1], [2], [8], [9]). The class $\mathfrak{C}$ has the following properties:

(1) CAT(0)-groups lie in $\mathfrak{C}$.

(2) Virtually solvable groups lie in $\mathfrak{C}$.

(3) The class $\mathfrak{C}$ is closed under taking subgroups.

(4) The class $\mathfrak{C}$ is closed under taking directed colimits.

(5) Let $f: G \rightarrow H$ be a group homomorphism and assume that $H$ lies in $\mathfrak{C}$ and $f^{-1}(C)$ lies in $\mathfrak{C}$ for any torsion-free cyclic subgroup $C$ of $H$. Then $G$ lies in $\mathfrak{C}$. 
Proof. For CAT(0)-groups, the version with finite wreath products follows from the version without wreath products, since the wreath product of a CAT(0)-group with a finite group is again a CAT(0)-group. The wreath product version for virtually solvable groups has been shown in [9, Theorem 1.1].

The inheritance properties of the version with finite wreath products follow easily from the inheritance properties of the version without wreath products; see for example [9, Proposition 2.17] and [9, Proposition 2.22].

We will individually refer to each property above as "property $(*)$. .

Corollary 2.2. If $G$ is a solvable group and $H \in \mathfrak{C}$ then any semidirect product $G \rtimes H$ lies in $\mathfrak{C}$.

Proof. Consider the projection homomorphism $G \rtimes H \rightarrow H$. The claim follows from properties (2) and (5), as preimages of cyclic subgroups of $H$ are solvable.

In particular, as finitely generated free groups are CAT(0)-groups, for any $m, n \in \mathbb{N}$ any semidirect product $\mathbb{Q}^{m} \rtimes F_{n}$ lies in $\mathfrak{C}$.

\section{Graphs of groups}

Given a connected graph $\Gamma$ (in the sense of Serre) and an oriented edge $e \in E(\Gamma)$, denote by $\iota(e) \in V(\Gamma)$ its initial and by $\tau(e) \in V(\Gamma)$ its terminal vertex. If by $\bar{e}$ we denote the edge $e$ with opposite orientation then $\iota(\bar{e})=\tau(e)$ and $\tau(\bar{e})=\iota(e)$. A graph of groups structure $\mathcal{G}$ on $\Gamma$ consists of families of groups $\left(G_{v}\right)_{v \in V(\Gamma)}$ and $\left(G_{e}\right)_{e \in E(\Gamma)}$ satisfying $G_{\bar{e}}=G_{e}$ for all $e \in E(\Gamma)$ together with an injective group homomorphism $\alpha_{e}: G_{e} \hookrightarrow G_{\iota(e)}$ for each $e \in E(\Gamma)$. We call the pair $(\Gamma, \mathcal{G})$ a graph of groups.

Given a maximal tree $T$ in $\Gamma$, let $\pi_{1}(\Gamma, \mathcal{G}, T)$ be the group generated by the groups $G_{v}, v \in V(\Gamma)$ and the elements $e \in E(\Gamma)$ subject to the relations

(i) $\bar{e}=e^{-1}$ for all $e \in E(\Gamma)$;

(ii) $e \cdot \alpha_{\bar{e}}(s) \cdot \bar{e}=\alpha_{e}(s)$ for all $e \in E(\Gamma)$ and $s \in G_{e}$;

(iii) $e=1$ if $e \in E(T)$.

We call $\pi_{1}(\Gamma, \mathcal{G}, T)$ the fundamental group of $(\Gamma, \mathcal{G})$ relative to $T$. For each $v \in$ $V(\Gamma)$ the canonical map $G_{v} \rightarrow \pi_{1}(\Gamma, \mathcal{G}, T)$ turns out to be injective [3, Corollary 1.9]. The isomorphism type of $\pi_{1}(\Gamma, \mathcal{G}, T)$ does not depend on the choice of $T$ [7, Proposition 20], and we will often speak of the fundamental group of $(\Gamma, \mathcal{G})$ and denote it by $\pi_{1}(\Gamma, \mathcal{G})$. 
Example 3.1. Let $G=B S(p, q)=\left\langle x, t \mid t x^{p} t^{-1}=x^{q}\right\rangle$. Then $G$ is isomorphic to the fundamental group of a graph of groups with a single edge $e$ and vertex $v$, where $G_{v}=G_{e}=\langle x\rangle \cong \mathbb{Z}$ and $\alpha_{\bar{e}}=\left(x \mapsto x^{p}\right)$ and $\alpha_{e}=\left(x \mapsto x^{q}\right)$.

A subgraph of subgroups of a graph of groups $(\Gamma, \mathcal{G})$ is a graph of groups $\left(\Gamma^{\prime}, \mathcal{G}^{\prime}\right)$ such that $\Gamma^{\prime} \subseteq \Gamma$, for all $v \in V\left(\Gamma^{\prime}\right)$ and $e \in E\left(\Gamma^{\prime}\right)$ we have $G_{v}^{\prime} \leq G_{v}$ and $G_{e}^{\prime} \leq G_{e}$ respectively, and $\alpha_{e}^{\prime}=\alpha_{e \mid G_{e}^{\prime}}$ for all $e \in E\left(\Gamma^{\prime}\right)$. If $T^{\prime}$ and $T$ are maximal trees in $\Gamma^{\prime}$ and $\Gamma$ respectively such that $T^{\prime} \subseteq T$ then there is a natural group homomorphism $\pi_{1}\left(\Gamma^{\prime}, \mathcal{G}^{\prime}, T^{\prime}\right) \rightarrow \pi_{1}(\Gamma, \mathcal{G}, T)$ that maps for $v \in V\left(\Gamma^{\prime}\right)$ every $x \in G_{v}^{\prime}$ to $x \in G_{v}$ and every $e \in E\left(\Gamma^{\prime}\right)$ to $e \in E(\Gamma)$.

Lemma 3.2. Let $\left(\Gamma_{i}, \mathcal{G}_{i}\right)_{i \in I}$ be a directed system of graphs of groups with binary relation $\subseteq$ where $\left(\Gamma_{i}, \mathcal{G}_{i}\right) \subseteq\left(\Gamma_{j}, \mathcal{G}_{j}\right)$ if $\left(\Gamma_{i}, \mathcal{G}_{i}\right)$ is a subgraph of subgroups of $\left(\Gamma_{j}, \mathcal{G}_{j}\right)$. Moreover, let $\left(T_{i}\right)_{i \in I}$ be a directed system of corresponding maximal trees, i.e. $T_{i}$ is a maximal tree in $\Gamma_{i}$ for all $i \in I$ and $T_{i} \subseteq T_{j}$ whenever $\left(\Gamma_{i}, \mathcal{G}_{i}\right) \subseteq$ $\left(\Gamma_{j}, \mathcal{G}_{j}\right)$. Let

- $\Gamma=\bigcup_{i \in I} \Gamma_{i}$;

- $\mathcal{G}=\bigcup_{i \in I} \mathcal{G}_{i}$ be the graph of groups structure on $\Gamma$ whose vertex and edge groups are the unions of the vertex and edge groups of the $\mathcal{G}_{i}$ 's and where for $e \in E(\Gamma)$ and $s \in G_{e}$ we define $\alpha_{e}(s)$ by $\left(\alpha_{e}\right)_{i}(s) \in\left(G_{\iota(e)}\right)_{i} \leq G_{\iota(e)}$ whenever $e \in E\left(\Gamma_{i}\right)$ and $s \in\left(G_{e}\right)_{i}$;

- $T=\bigcup_{i \in I} T_{i}$ be our choice of a maximal tree in $\Gamma$.

Consider the directed system of fundamental groups of graphs of groups defined by the natural maps $\pi_{1}\left(\Gamma_{i}, \mathcal{G}_{i}, T_{i}\right) \rightarrow \pi_{1}\left(\Gamma_{j}, \mathcal{G}_{j}, T_{j}\right)$ whenever $\left(\Gamma_{i}, \mathcal{G}_{i}, T_{i}\right) \subseteq$ $\left(\Gamma_{j}, \mathcal{G}_{j}, T_{j}\right)$. We have

$$
\pi_{1}(\Gamma, \mathcal{G}, T) \cong \operatorname{colim}_{i \in I} \pi_{1}\left(\Gamma_{i}, \mathcal{G}_{i}, T_{i}\right) .
$$

Proof. It easily follows from the definition of $(\Gamma, \mathcal{G}, T)$ that $\pi_{1}(\Gamma, \mathcal{G}, T)$ has the universal property of $\operatorname{colim}_{i \in I} \pi_{1}\left(\Gamma_{i}, \mathcal{G}_{i}, T_{i}\right)$, whence the claim.

Given a graph of groups $(\Gamma, \mathcal{G})$, one can define a simplicial tree $X=\widetilde{(\Gamma, \mathcal{G}})$, the Bass-Serre covering of $(\Gamma, \mathcal{G})$, and a continuous map $p: X \rightarrow \Gamma$ sending edges to edges such that the group $\pi_{1}(\Gamma, \mathcal{G})$ acts on $X$ by simplicial automorphisms without edge inversions and the stabilizer of $v \in V(X)$ is conjugate to the vertex group $G_{p(v)} \in \mathcal{G}$. Vice versa, by the fundamental theorem of Bass-Serre theory [7, section I.5.3] any action of a group $G$ on a simplicial tree $T$ gives rise to a (generally non-canonical) graph of groups structure $\mathcal{G}$ on the quotient graph $G \backslash T$ such that $\pi_{1}(G \backslash T, \mathcal{G}) \cong G$. 
Lemma 3.3. If a group acts on a simplicial tree with finite point stabilizers then it lies in $\mathfrak{C}$.

Proof. A group that acts on a simplicial tree with finite point stabilizers is isomorphic to the fundamental group of a graph of finite groups $(\Gamma, \mathcal{G})$. By Lemma 3.2, the group $\pi_{1}(\Gamma, \mathcal{G})$ is isomorphic to the colimit of the directed system of fundamental groups associated to the directed system of finite subgraphs of subgroups of $(\Gamma, \mathcal{G})$. Fundamental groups of finite graphs of finite groups are CAT $(0)$-groups (in fact, they are virtually finitely generated free) and hence lie in $\mathfrak{C}$ by property (1). Therefore, $\pi_{1}(\Gamma, \mathcal{G})$ is isomorphic to the colimit of a directed system of groups that lie in $\mathfrak{C}$ and hence lies in $\mathfrak{C}$ by property (4).

A tree of groups is a graph of groups whose underlying graph is a tree.

Proposition 3.4. The fundamental group of a finite tree of finitely generated abelian groups $(T, \mathcal{G})$ is a $\mathrm{CAT}(0)$-group.

We will make use of the following theorem:

Theorem 3.5 (Equivariant Gluing [4, II.11.18]). Let $\Gamma_{0}, \Gamma_{1}$ and $H$ be groups acting properly by isometries on complete $\mathrm{CAT}(0)$ spaces $X_{0}, X_{1}$ and $Y$ respectively. Suppose that for $j=0,1$ there exists both an injective group homomorphism $\varphi_{j}: H \rightarrow \Gamma_{j}$ and a $\varphi_{j}$-equivariant isometric embedding $f_{j}: Y \rightarrow X_{j}$. Then

(1) the amalgamated free product $\Gamma=\Gamma_{0} *_{H} \Gamma_{1}$ associated to the maps $\varphi_{j}$ acts properly by isometries on a complete $\mathrm{CAT}(0)$ space $X$;

(2) if the given actions of $\Gamma_{0}, \Gamma_{1}$ and $H$ are cocompact, the action of $\Gamma$ on $X$ is cocompact.

We also need that the spaces $X_{0}$ and $X_{1}$ embed equivariantly and isometrically into $X$. However, this is clear from the construction given in the proof of the Equivariant Gluing Theorem in [4].

Proof of Proposition 3.4. Define for $v \in V(T)$ and $e \in E(T)$ the R-vector spaces $X_{v}=G_{v} \otimes_{\mathbb{Z}} \mathbb{R}$ and $X_{e}=G_{e} \otimes_{\mathbb{Z}} \mathbb{R}$ respectively. The induced action of $G_{v}$ on $X_{v}$ given by

$$
\begin{aligned}
G_{v} \times X_{v} & \longrightarrow X_{v}, \\
(g, x) & \longmapsto(g \otimes 1)+x,
\end{aligned}
$$


is proper and cocompact, and we analogously obtain a proper and cocompact action of $G_{e}$ on $X_{e}$. Let $v_{0} \in V(T)$ and exhaust the finite tree $T$ by subtrees

$$
\left\{v_{0}\right\}=T_{0} \subset \cdots \subset T_{n}=T
$$

such that for all $i=1, \ldots, n$ the tree $T_{i}$ has one more vertex $v_{i}$ than $T_{i-1}$. We will denote the graph of groups structure on $T_{i}$ obtained by restricting $\mathcal{G}$ to $T_{i} \subseteq T$ also by $\mathcal{G}$. For each $i=1, \ldots, n$ denote by $e_{i}$ the unique oriented edge of $T_{i}$ for which $\iota\left(e_{i}\right) \in V\left(T_{i-1}\right)$ and $\tau\left(e_{i}\right)=v_{i}$.

Choose an inner product on the finite-dimensional $\mathbb{R}$-vector space $X_{v_{0}}$ and thereby endow it with a complete CAT( 0$)$ metric. Independent of this choice, $G_{v_{0}}$ acts on $X_{v_{0}}$ by isometries. We inductively construct for each $i=1, \ldots, n$ inner products on $X_{e_{i}}$ and $X_{v_{i}}$ such that the $\alpha_{e_{i}}$-equivariant respectively $\alpha_{\overline{e_{i}}}$-equivariant embeddings

$$
X_{\iota\left(e_{i}\right)} \longleftrightarrow X_{e_{i}} \longleftrightarrow X_{v_{i}}
$$

induced by the injective edge homomorphisms

$$
\pi_{1}\left(T_{i-1}, \mathcal{G}\right) \geq G_{l\left(e_{i}\right)} \stackrel{\alpha_{e_{i}}}{\longleftarrow} G_{e_{i}} \stackrel{\alpha_{\overline{e_{\bar{i}}}}}{\longrightarrow} G_{v_{i}}
$$

are isometric. In order to do so, pull back the inner product on $X_{l\left(e_{i}\right)}$ to obtain an inner product on $X_{e_{i}}$. Then, choose any inner product on $X_{v_{i}}$ that extends the inner product on $X_{e_{i}} \hookrightarrow X_{v_{i}}$.

By applying Theorem 3.5 repeatedly, we construct for $i=1, \ldots, n$ a complete CAT( 0$)$ space $X_{T_{i}}$ on which $\pi_{1}\left(T_{i}, \mathcal{G}\right)$ acts properly and cocompactly by isometries, and into which for $j \leq i$ each $X_{v_{j}}$ embeds equivariantly and isometrically.

Remark. Free products with amalgamation of virtually finitely generated abelian groups need not be CAT(0)-groups; for a counterexample, see [4, III.Г.6.13]. However, if the amalgam is virtually cyclic then the vertex groups can be arbitrary CAT(0)-groups [4, Corollary II.11.19].

Corollary 3.6. The fundamental group of a tree of finitely generated abelian groups lies in $\mathfrak{C}$.

Proof. Any graph of groups can be exhausted by the directed system of its finite subgraphs of groups. The claim follows from Lemma 3.2, Proposition 3.4, and properties (1) and (4). 


\section{Proof of the main theorem}

Proof of the Main Theorem. We may assume that $(\Gamma, \mathcal{G})$ is a finite graph of finitely generated abelian groups; this follows from three consecutive applications of Lemma 3.2.

(1) Let $(\Gamma, \mathcal{G})$ be a finite graph of abelian groups with finitely generated edge groups. For every vertex $v \in V(\Gamma)$ there exists a finitely generated subgroup of $G_{v}$ that contains the images of all adjacent edge homomorphisms so that we can easily find a directed system of finite subgraphs of finitely generated subgroups of $(\Gamma, \mathcal{G})$ that exhausts $(\Gamma, \mathcal{G})$.

(2) If $(\Gamma, \mathcal{G})$ is a finite graph of abelian groups, we can write every edge group as the directed colimit of its finitely generated subgroups and $\pi_{1}(\Gamma, \mathcal{G})$ as the directed colimit of fundamental groups of finite graphs of abelian groups with finitely generated edge groups.

(3) Finally, any graph of (abelian) groups can be exhausted by the directed system of its finite subgraphs of (abelian) groups.

Let $T$ be a maximal tree in $\Gamma$. We will construct a group homomorphism $\phi$ from $\pi_{1}(\Gamma, \mathcal{G}, T)$ to a group of the form $\mathbb{Q}^{m} \rtimes F_{n}$, where $\mathbb{Q}^{m} \rtimes F_{n}$ lies in $\mathfrak{C}$ by Corollary 2.2. We then prove that $\pi_{1}(\Gamma, \mathcal{G}, T)$ lies in $\mathfrak{C}$ by showing that all preimages of torsion-free cyclic subgroups of $\mathbb{Q}^{m} \rtimes F_{n}$ lie in $\mathfrak{C}$, i.e. by applying property (5).

Let the vertex set of $\Gamma$ be given by $\left\{v_{1}, \ldots, v_{k}\right\}$ and define $Q$ as the $\mathbb{Q}$-vector space

$$
Q=\bigoplus_{i=1}^{k}\left(G_{v_{i}} \otimes_{\mathbb{Z}} \mathbb{Q}\right) .
$$

For every $e \in E(\Gamma)$ the injective group homomorphism $\alpha_{e}: G_{e} \rightarrow G_{\iota(e)}$ gives rise to an injective $\mathrm{Q}$-linear homomorphism

$$
M_{e}=\alpha_{e} \otimes_{\mathbb{Z}} \mathrm{id}: G_{e} \otimes_{\mathbb{Z}} \mathbb{Q} \longrightarrow G_{\iota(e)} \otimes_{\mathbb{Z}} \mathbb{Q} .
$$

Define $R$ to be the $\mathrm{Q}$-subvector space of $Q$ spanned by the vectors

$$
M_{\bar{e}}(s \otimes 1)-M_{e}(s \otimes 1) \quad \text { for all } e \in E(T), s \in G_{e} .
$$

For every vertex $v \in V(\Gamma)$ the rationalized vertex group $G_{v} \otimes_{\mathbb{Z}} \mathbb{Q}$ naturally embeds into $Q / R$, which can be seen as follows: Fix an orientation $\mathcal{O}(T) \subset E(T)$ for 
each edge of $T$ and suppose that this is not the case, i.e. we can find an element $0 \neq q \in G_{v} \otimes_{\mathbb{Z}} \mathbb{Q}$ and for every $e \in \mathcal{O}(T)$ an element $q_{e} \in G_{e} \otimes_{\mathbb{Z}} \mathbb{Q}$ such that

$$
q=\sum_{e \in \mathcal{O}(T)}\left(M_{\bar{e}}\left(q_{e}\right)-M_{e}\left(q_{e}\right)\right) \in Q .
$$

Consider the subforest $F \subseteq T$ spanned by all edges for which $q_{e} \neq 0$. It contains at least one edge, as the right hand side of (4.1) would otherwise be zero, contradicting that $q \neq 0$. Choose a leaf $w \in V(F)$, i.e. a vertex of valence 1 , such that $w \neq v$ and let $e$ be the unique edge in $\mathcal{O}(T) \cap E(F)$ adjacent to $w$, say with $\iota(e)=w$. Since $q \in G_{v} \otimes_{\mathbb{Z}} \mathbb{Q}$ with $v \neq w$ and $e$ is the only edge in $\mathcal{O}(T) \cap E(F)$ adjacent to $w$, projecting (4.1) to the factor $G_{w} \otimes_{\mathbb{Z}} \mathbb{Q} \leq Q$ gives rise to the equation

$$
0=-M_{e}\left(q_{e}\right) .
$$

However, this is a contradiction, as $q_{e} \neq 0$ and $M_{e}$ is injective.

Let $\left\{e_{1}, \ldots, e_{n}\right\}$ be the set of edges of $\Gamma \backslash T$ and denote by $F_{n}$ the free group with basis $\left\{e_{1}, \ldots, e_{n}\right\}$. We obtain a linear representation

$$
\rho: F_{n} \longrightarrow \mathrm{GL}(Q / R)
$$

by extending for every $e_{i}$ the isomorphism of subspaces

$$
M_{e_{i}} \circ M_{\bar{e}_{i}}^{-1}: \operatorname{im}\left(M_{\overline{e_{i}}}\right) \stackrel{\cong}{\longrightarrow} \operatorname{im}\left(M_{e_{i}}\right)
$$

to an automorphism of the finite-dimensional $\mathrm{Q}$-vector space $Q / R$. Define a group homomorphism

$$
\phi: \pi_{1}(\Gamma, \mathcal{G}, T) \longrightarrow(Q / R) \rtimes_{\rho} F_{n}
$$

by mapping for $v \in V(\Gamma)$ any element $x \in G_{v}$ to $(x \otimes 1,1)$ and $e \in E(\Gamma \backslash T)$ to $(0 \otimes 0, e)$. This assignment is well-defined: Suppose that $x \in G_{v}$ lies in the image of $\alpha_{e}$ for some edge $e \in E(\Gamma)$ with $\iota(e)=v$, i.e. $x=\alpha_{e}(s)$ for some $s \in G_{e}$. Then $\phi(x)=\phi\left(\alpha_{e}(s)\right)=\left(\alpha_{e}(s) \otimes 1,1\right)$. By definition, in $\pi_{1}(\Gamma, \mathcal{G}, T)$ we have that

$$
x= \begin{cases}\alpha_{\bar{e}}(s) & \text { if } e \in E(T), \\ e \cdot \alpha_{\bar{e}}(s) \cdot \bar{e} & \text { if } e \in E(\Gamma \backslash T) .\end{cases}
$$

In the first case, $\phi\left(\alpha_{\bar{e}}(s)\right)=\left(\alpha_{\bar{e}}(s) \otimes 1,1\right)$, where $\alpha_{\bar{e}}(s) \otimes 1=\alpha_{e}(s) \otimes 1$ in $Q / R$ and hence $\phi\left(\alpha_{\bar{e}}(s)\right)=\phi(x)$. In the second case,

$$
\begin{aligned}
\phi\left(e \cdot \alpha_{\bar{e}}(s) \cdot \bar{e}\right) & =(0 \otimes 0, e) \cdot\left(\alpha_{\bar{e}}(s) \otimes 1,1\right) \cdot(0 \otimes 0, \bar{e}) \\
& =\left(0 \otimes 0+\rho(e)\left(\alpha_{\bar{e}}(s) \otimes 1\right)+0 \otimes 0, e \bar{e}\right) \\
& =\left(M_{e}\left(M_{\bar{e}}^{-1}\left(\alpha_{\bar{e}}(s) \otimes 1\right)\right), e \bar{e}\right) \\
& =\left(\alpha_{e}(s) \otimes 1,1\right)=\phi(x)
\end{aligned}
$$


whence $\phi$ is well-defined. Recall that $(Q / R) \rtimes_{\rho} F_{n}$ lies in $\mathfrak{C}$ by Corollary 2.2, $Q / R$ being isomorphic to $\mathbb{Q}^{m}$ for some $m \in \mathbb{N}$.

Let $C$ be a torsion-free cyclic subgroup of $(Q / R) \rtimes_{\rho} F_{n}$ and first assume that $C$ is not contained in $(Q / R) \rtimes\{1\}$. Consider the induced subgroup action of $\phi^{-1}(C) \leq \pi_{1}(\Gamma, \mathcal{G}, T)$ on the Bass-Serre covering tree $X=(\Gamma, \mathcal{G})$ and recall that every point stabilizer of this action is contained in a conjugate of some vertex group $G_{v}, v \in V(\Gamma)$. For each vertex $v \in V(\Gamma)$ the kernel of the natural map $G_{v} \rightarrow G_{v} \otimes_{\mathbb{Z}} \mathbb{Q}$ equals the torsion subgroup of $G_{v}$, and $G_{v} \otimes_{\mathbb{Z}} \mathbb{Q}$ embeds into $Q / R$ and hence into the normal subgroup $(Q / R) \rtimes\{1\}$. Consequently, $\phi^{-1}(C)$ contains of every point stabilizer only its torsion subgroup and acts on $X$ with finite point stabilizers. We conclude that $C$ lies in $\mathfrak{C}$ by Lemma 3.3.

On the other hand, if $C$ is contained in $(Q / R) \rtimes\{1\}$, consider the composition of group homomorphisms

$$
\Phi: \pi_{1}(\Gamma, \mathcal{G}, T) \stackrel{\phi}{\longrightarrow}(Q / R) \rtimes_{\rho} F_{n} \longrightarrow F_{n}
$$

where the second homomorphism is given by projection to the second factor. The preimage $\phi^{-1}(C)$ is a subgroup of $\operatorname{ker}(\Phi)$, whence, by property (3), in order to show that $\phi^{-1}(C)$ lies in $\mathfrak{C}$ it suffices to show that $\operatorname{ker}(\Phi)$ lies in $\mathfrak{C}$. We claim that $\operatorname{ker}(\Phi)$ is isomorphic to the fundamental group of a tree of finitely generated abelian groups and hence lies in $\mathfrak{C}$ by Corollary 3.6. Equivalently, we claim that $\operatorname{ker}(\Phi)$ acts on a tree with finitely generated abelian point stabilizers and contractible quotient. Since $\pi_{1}(\Gamma, \mathcal{G}, T)$ acts on $X$ with finitely generated abelian point stabilizers, it suffices to show that the quotient $\operatorname{ker}(\Phi) \backslash X$ is a tree. Note that $\pi_{1}(\Gamma, \mathcal{G}, T) / \operatorname{ker}(\Phi) \cong F_{n}$ and thus we obtain an induced action of the free group $F_{n}$ on $\operatorname{ker}(\Phi) \backslash X$. As every point stabilizers of the $\pi_{1}(\Gamma, \mathcal{G}, T)$-action on $X$ is contained in $\operatorname{ker}(\Phi)$, the action of $F_{n}$ on $\operatorname{ker}(\Phi) \backslash X$ is free. We conclude that $\operatorname{ker}(\Phi) \backslash X$ is the universal covering space of the finite graph $F_{n} \backslash(\operatorname{ker}(\Phi) \backslash X) \cong \Gamma$ and therefore a tree.

\section{References}

[1] A. Bartels, T. Farrell, and W. Lück, The Farrell-Jones conjecture for cocompact lattices in virtually connected Lie groups. J. Amer. Math. Soc. 27 (2014), no. 2, 339-388. Zbl 1307.18012 MR 3164984

[2] A. Bartels and W. Lück, The Borel conjecture for hyperbolic and CAT(0)-groups. Ann. of Math. (2) 175 (2012), no. 2, 631-689. Zbl 1256.57021 MR 2993750

[3] H. Bass, Covering theory for graphs of groups. J. Pure Appl. Algebra 89 (1993), no. 1-2, 3-47. Zbl 0805.57001 MR 1239551 
[4] M. R. Bridson and A. Haefliger, Metric spaces of non-positive curvature. Grundlehren der Mathematischen Wissenschaften, 319. Springer, Berlin, 1999. Zbl 1744486 MR 0988.53001

[5] T. Farrell and X. Wu, Farrell-Jones conjecture for the solvable Baumslag-Solitar groups. Math. Ann. 359 (2014), no. 3-4, 839-862. Zbl 06323294 MR 3231018

[6] W. Lück and H. Reich, The Baum-Connes and the Farrell-Jones conjectures in Kand L-theory. In E. M. Friedlander and D. R. Grayson (eds.), Handbook of K-theory. Vol. 2. Springer, Berlin, 2005, 703-842. Zbl 1120.19001 MR 2181833

[7] J.-P. Serre, Trees. Translated from the French by J. Stillwell. Springer, Berlin etc., 1980. Zbl 0548.20018 MR 0607504

[8] Ch. Wegner, The K-theoretic Farrell-Jones conjecture for CAT(0)-groups. Proc. Amer. Math. Soc. 140 (2012), no. 3, 779-793. Zbl 1240.19003 MR 2869063

[9] Ch. Wegner, The Farrell-Jones conjecture for virtually solvable groups. Preprint 2013. arXiv:1308.2432 [math.GT]

Received October 3, 2013

Giovanni Gandini, Institut for Matematiske Fag, Københavns Universitet, Universitetsparken 5, 2100 København Ø, Denmark

home page: http://www.math.ku.dk/ zjb179

e-mail: ggandini@math.ku.dk

Sebastian Meinert, Freie Universität Berlin, Institut für Mathematik, Arnimallee 7, 14195 Berlin, Germany

home page: http://meinert-online.de/personal

e-mail: sebastian.meinert@gmail.com

Henrik Rüping, Department of Mathematics, The University of British Columbia, 1984 Mathematics Road, Vancouver, B.C., Canada V6T 1 Z2

home page: http://www.math.uni-bonn.de/people/rueping

e-mail: rueping@math.ubc.ca 